\title{
Instructional simulation: The interface with the student
}

\author{
RICHARD R. JOHNSON* \\ Exxon Education Foundation, New York, New York 10020
}

\begin{abstract}
Several significant issues relating to the interface of simulation models with the student are discussed, and suggestions are made for new directions in development.
\end{abstract}

The papers in this symposium have described a group of data-generating computer programs which have been developed over the last 4 years for use in instruction on experimentation in psychology. The programs described have progressed a long way in their sophistication from the first crude model I tested in 1968. However, the development of instructional uses of computer simulation goes back further than these recent experiments and, more significantly, has a long way to go.

Probably the earliest instructional uses of computer simulation involved models built for research that were used as demonstration devices by allowing students to "look over the shoulder" of an individual manipulating a model which had not been designed for instruction. An example of this use of simulation in psychology was described by Hoffman (1962). He reported on an electronic black box, called the "analogue lab," which was influenced in its design by earlier models built in Hull's laboratory in the late 1920s and 1930s. Though not recognized as such, this was, in effect, a special-purpose analogue computer built to demonstrate a variety of variables in classical conditioning. Examples of experimental simulations presented in this symposium represent a significant advance in both breadth and complexity of the experimental problems that can be simulated. And there is no reason to doubt that this technique can be extended into newer areas through the development of even more sophisticated models. There is, however, a very significant question that we need to consider more systematically before we become too caught up in moving toward more elegant computer programs and more refined models. If we intend to use simulations as instructional devices, we need to know more about their interface with the student. The first euphoric proliferation of CAI seems now to be giving way to more interest in the careful design of the instructional system focusing heavily on this interface (e.g., Atkinson, 1972). I am convinced that the same must be true with instructional simulation: the millennium in the laboratory has not come with our more elegant computer models; rather, we need to settle

\footnotetext{
*Reprint requests should be mailed to the author, c/o Exxon Education Foundation, 111 West 49th Street, New York, New York 10020.
}

down to tackle several very significant issues relating to the interface of these models with the student. I intend, in this paper, to outline several of these issues which need to be studied, and to present some suggestions for new directions in development.

\section{HOW GOOD IS A PARTICULAR SIMULATION MODEL AS A TEACHING DEVICE?}

As the library of available computer simulations continues to enlarge, it will be important for us to determine how good a particular simulation is as an instructional device in relation to others, or the standard instructional methods which might be used in its place. More specifically, we will need to consider how good a particular simulation is for a given purpose in order to prescribe the appropriate simulated problem for students needing to develop particular skills.

Obviously, we will need to continue attempts to evaluate subject matter gains through simulated experimentation as well as assessing the development of skills in experimental design. In addition, a major focus of our evaluation should be on assessing transfer of skills from simulated learning to real experimentation. I would like to suggest two other ways in which simulation models could be examined as instructional devices.

The fact that all the experimental design decisions of students interacting with a computer-based model can be stored for later analysis provides a wealth of data on successive decision-making during the learning process. While I made initial attempts to analyze these data at Earlham College, the very small $\mathrm{N}$ available provided only the simplest possible categorization of different student research strategies. As the extant simulations are run successively with large classes, there is the potential for amassing a wealth of information concerning how student experimental design decisions change as a function of the information they receive on earlier rounds of simulated experimentation. Under these conditions, it should be possible to develop student norms of decision strategy for particular computer models and to examine how student development of research skill varies across different models. In this way, the utility of our simulations could be examined by noting how developmental sequences vary rather than by 
merely examining end-point outcomes.

A second approach to developing norms for simulation models involves the use of criterion reference groups. I made an attempt at this approach to norm development by mailing instructions for interacting with one of my DATACALL simulations to a group of experimental psychologists about 2 years ago. To rule out any advantage they might have in knowledge of the subject matter, I did not label any of the variables in the model and asked them to play this "black-box" game to determine which variables were important in producing the outcomes. Under these conditions, one can examine research strategy without the bias of prior knowledge of the subject matter. I am happy to say that there were marked differences in the way experimental psychologists interact with the simulation as opposed to my freshman students. If we can assume that the research designs developed by experimental psychologists under conditions of minimal knowledge represent optimal strategies, then we can examine student decision patterns to see whether they begin to approximate these criterion strategies as a result of interacting with the simulation over a period of time. Again, we have a chance to examine a particular simulation model to see if it tends to evoke these optimal strategies as rapidly and as efficiency as possible.

Another approach to evaluation of simulations that 1 began to examine at Earlham involved a mathematical game theory analysis of the computer model. All of the simulations presently existing (in their mathematical modeling and rules for use in the laboratory) have solutions in the mathematical game-theory sense. That is, if we conceive of the situation presented to the student in the simulated laboratory as a game in which he interacts with the computer to gain certain ends, then we can specify definitively what his rational strategy should be. Specifically, if an optimum outeome is specified and the restraints on experimental design indicated, knowing the distribution of effects of variables in the computer model, we can determine his best strategy. This appears to be a linear programming problem that could be readily handled by developing another computer program. This second game-analyzer program might interact with any computer simulation given to it, as an "idealized, simulated student," to show an instructor precisely what rational student research behavior would look like for the model he has developed. Under these conditions, it should be relatively easy to compare two simulations, before any students ever interact with them, as to the rational research behavior they should evoke.

\section{HOW CAN WE FINE-TUNE COMPUTER SIMULATIONS AS TEACHING DEVICES?}

A number of the dimensions on which computer simulation models may vary need to be examined far more carefully to ascertain their influence on instruction. The subject matter, the fidelity of the model to the area simulated, and the relative complexity of the model need to be considered in their effects upon bringing forth good research strategy from beginning students. In addition, we need to pay far more attention to the "stimulus value" of our computer simulations. To what extent do certain models succeed in evoking more complex experimental designs? What kinds of models seem to goad students into studying statistics on their own or interacting with other students in attempting to learn research design? What types of simulations stimulate students to "cheat" by going to the library to read up on the problem area considered? If we can begin to understand the sharacteristics of those simulations which lead to these kinds of student behaviors, we may be able to fine-tune our laboratory materials to optimize research training. Certainly, the attempts to develop normative data on the models, mentioned above, should also belp us to adjust the models to meet instructional goals. In the same way, an instructor who has a "game analyzer" program available for testing the simulation he has designed should be able to look at its implications and fine-tune the model to achieve a predicted rational design behavior before students interact with his simulation.

The preceding suggestions for modification of the computer-student interface have all focused on ways of changing the computer programs. Perhaps more significant adjustments may be effected by focusing on the instructional context within which the computer simulation is used. Let me begin by asserting that our present instructional environments are artificial. The activities occurring and the "demand characteristics" of our laboratories as well as our lecture halls correspond very poorly to any "real world" situations. The student who is assigned an experiment to run for which there is already a known answer and who is handed a list of steps to be followed in carrying out the experiment is not behaving like a scientist, even though he may personally handle a Skinner box or wear a white laboratory coat. Recognizing that the entire laboratory experience has been in some sense a simulation, we need to ask how this training period can best be designed to maximize learning and transfer. I am a strong advocate of using these computer simulations within the context of games. Allow me to attempt to rationalize this heresy.

A student is allowed a great deal of freedom to engage in open-ended problem solving at a low risk via computer simulations. However, it is obviously counterproductive to have him think that research involves random patterns of questions within a finite problem environment to produce an easy return of data. Even though our computer models will allow it, we do not want students to set up a separate experimental group for all potential variables or ask for a thousand pieces of data for each cell. A relatively easy way of setting up constraints on the use of any computer model is to attach a cost, in points, to each of the decisions 
involved in an experimental design. Under these conditions, the student must pay for each experiment run in relation to the complexity of the design. A payoff in points may then be returned for the student's laboratory report ("publication") when it is turned in. If the payoff varies with the amount of information returned in the study, i.e., the number and kinds of conclusions the student is able to form, then he will be biased toward running the most efficient experiments which return the greatest amount of information.

This game environment has a number of distinct advantages. First, the instructor has set up explicit, but generalized, goals for the entire class. As a corollary to this, at any time during the course of his "experimentation," a student knows how he is doing by noting his point return. In addition, I have found that a variety of other interesting student behaviors are emitted in the game environment. When student lab reports are posted in a public place, I have seen students testing the conclusions of other students by replicating and refining prior studies. In this fashion, the self-corrective nature of scientific research can be mirrored. In addition, the cost-payoff structure of the game environment has led some students to "discover" the utility of pilot studies, while others have develped reduced experimental designs. Higher payoffs for greater information returns lead to the designing of more complex experiments, and students are then motivated to learn more sophisticated techniques of data analysis, such as analysis of variance. At least a dozen different game structures have already been described (Johnson, 1973), and certainly many more wait to be invented. By combining the carefully designed computer simulation with an equally well-designed context, the instructor has a great deal of control over the demand characteristics of the learning environment.

This paper has been less a report on the use of data-generating computer models than it has been a polemic for certain modes of their development and use. I make no apologies, since I believe that, in talking about the instructional uses of the computer, after we have discussed hardware and reported on new software developments, we still need to consider pedagogy. In speaking to a group of computer buffs, this need is perhaps even greater. I have no doubt that more computer simulations will be written. I am concerned, however, that in our striving to produce more elegant models we not lose sight of the student, who is, after all, a part of the instructional system. It is for this reason that I have chosen to emphasize this interface. While some of the suggestions that I have made may sound radical in relation to our past practice in laboratory instruction, I feel strongly that some of these experiments need to be attempted and that the computer-simulated experiment offers an ideal medium for this experimentation. If we reject such radical proposals as playing games in the psychology laboratory, I hope it will not be on the basis of habit and tradition. We urge our undergraduate laboratory students to test the propositions of psychology empirically: these suggestions concerning instructional design deserve the same treatment.

\section{REFERENCES}

Atkinson, R. C. Ingredients for a theory of instruction. American Psychologist, 1972, 27, 921-931.

Hoffman, H. S. The analog lab: A new kind of teaching device. American Psychologist, 1962, 17, 684-694.

Johnson, R. R. DATACALL: What it is and how to do it. Unpublished descriptive materials available from Earlham College, Richmond, Indiana, 1973. 